\title{
VARIABLE STRUCTURE UNIT VECTOR CONTROL OF ELECTRIC POWER GENERATION
}

\author{
F. N. Okafor \\ Electrical/Electronics Engineering Department \\ University of Lagos, Nigeria \\ E-mail: Pcnwilo@infoweb.abs.net
}

\begin{abstract}
A variable structure Automatic Generation Control (VSAGC) scheme is proposed in this paper for the control of a single area power system model dominated by steam powered electric generating plants. Unlike existing, VSAGC scheme where the selection of the control function is based on a trial and error procedure, the strategy proposed here relies on a systematic approach based on the unit vector control concept. Simulation results show the superiority of the proposed control scheme over the existing ones.
\end{abstract}

\section{INTRODUCTION}

The application of variable structure control schemes to the solution of the automatic generation control (AGC) problem in power systems has been investigated at various times [1 - 4]. However the design of variable structure control systems (VSCS) basically involves two steps viz. $[5-9]$.

The design of the sliding plane to ensure stability of motion towards the origin of coordinates otherwise referred to as the existence problem. While sliding the system acquires certain desirable properties such as insensitivity to some class of parameter variations and extraneous disturbances.

ii) The selection of discontinuous control functions together with appropriate switching logic so that the system state is driven from an arbitrary initial condition and hastened to the sliding plane where it continues towards the origin. This is also referred to as the reachability problem.

In a pioneer work the authors of reference [1] successfully applied the variable structure control concept to the generation control of a single area power system model dominated by steam powered generating sets of the non-reheat type. Though the results are encouraging, the design procedure calls for some improvement. For instance, in constructing the sliding hyperplane an approach based on satisfying the Itkins condition [5] was adopted. Furthermore, their choice of discontinuous control functions consisted of relays with state dependent gains [6]. Either way, the determination of appropriate values of the gain coefficients both for the sliding equation and the control functions is fundamentally a trial and error procedure and could be time consuming.

Meanwhile, by employing a particular canonical transformation, Utkin and Yang [7] was able to formulate the design of a stable sliding mode as a linear state feedback control problem. Thus a solution can be found using any of the well-known design techniques of linear regulator theory such as pole assignment or optimal control scheme. Based on the above result, the authors of $[2,3]$ employed a procedure derived from optimal linear regulator theory for the synthesis of stable sliding mode for the Variable Structure Automatic Generation Controller (VSAGC) reported there in. Similarly an algorithm based on pole placement technique for linear systems is adopted in [4] for sliding mode synthesis. Both approaches are systematic and elegant. However, the same trial and error procedure as in [1] is employed in [2, 3] and [4] for the selection of the control functions. This is a major drawback for which an improvement is desirable.

In this paper therefore, a variable structure unit vector control scheme is proposed for a single power system model dominated by steam powered generators with reheat turbines as used in [2]. However the scheme presented here is unique in adopting a systematic procedure based on the unit vector control algorithm [ 9 ], for the synthesis of the control functions. An analysis using Lyapunnov's direct method ensures stability of motion towards the sliding hyperplane. 
Section 2 describes the system model while the automatic generation control problem is explained in section 3 . In section 4 , the synthesis procedure for the variable structure controller is discussed and then applied to the AGC problem in section 5. Section 6 presents some concluding remarks.

\section{Power System Model}

The objective of automatic generation control for an isolated area is to maintain a match between power generation and consumption so as to retain the frequency at its nominal value. Therefore the power system is modeled by its power balance equation, which relates the disparity between power generated and power consumed to the change in accelerating power and frequency. Assuming first order load perturbations, which allow for incremental linear model dynamics of the power-system load-frequency interaction, the standard analytical representation may be in the form of block diagram of fig. 1. This is the same model employed by Chan and Hsu [2] and is adopted here for easy comparison of results. The block diagram represents a single area power system dominated by steam powered plants with reheat turbines. The turbine dynamics are modelled by two first order time lags with time constants $\mathrm{T}_{\mathrm{rh}}$ and $\mathrm{T}_{\text {ch }}$, respectively and reheat coefficient $\mathrm{F}_{1}$. The load - frequency dynamics of the power system are modelled by a first order time lag characterised by time constant, $\mathrm{T}_{\mathrm{p}}$, and gain constant $\mathrm{K}_{\mathrm{p}}$ while the time lag due to the governing system as well as its feedback effect are represented by time constant $T_{G}$ and gain $1 / R$ respectively. The block marked (1/s) represents an additional integrator, which serves to ensure zero steady state frequency deviation.

Finally the variable structure control block is labeled VSC and the output is the control signal $\mathrm{u}=\Delta \mathrm{P}_{\mathrm{c}}$. The system model can be written in the state variable form as:
$X(t)=A x(t)+B u(t)+\Gamma \xi(t) ; x(0)=-x_{s s}$

Where x $\varepsilon \mathfrak{R}^{5}=\left(\int \Delta\right.$ f.dt $\left.\Delta \mathrm{x}_{\mathrm{e}} \Delta \mathrm{f} \quad \Delta \mathrm{P}_{\mathrm{G}} \Delta \mathrm{X}_{\mathrm{r}}\right)$

With $\mathrm{x}_{\mathrm{ss}} \rightarrow$ the nominal steady - state value

$\int \Delta \mathrm{f} . \mathrm{dt} \rightarrow$ load angle deviation from the nominal value

$$
\Delta \mathrm{x}_{\mathrm{e}} \rightarrow \text { change in turbine valve position (pu) }
$$

$\Delta \mathrm{f} \rightarrow$ frequency deviation $(\mathrm{Hz})$

$\Delta \mathrm{P}_{\mathrm{G}} \rightarrow$ change in generated power $(\mathrm{pu})$

$\Delta \mathrm{X}_{\mathrm{r}} \rightarrow$ pu change in reheat output power.

The control signal $\mathrm{u}=\Delta \mathrm{P}$ is a measure of the deviation in speed changer position while the disturbance signal $=\Delta P_{D}$ represents a step change in load demand. The system matrix A $\varepsilon \mathfrak{R}^{5 \times 5}$, the control matrix $\mathrm{B} \varepsilon \mathfrak{R}^{5 \times 1}$ and the disturbance matrix $\Gamma \varepsilon \mathfrak{R}^{5 \times 1}$ are defined as follows:

$$
\begin{aligned}
& \mathrm{A}=\left[\begin{array}{ccccc}
0 & 0 & 1 & 0 & 0 \\
0 & \frac{-1}{\mathrm{~T}_{\mathrm{G}}} & \frac{-1}{\mathrm{~T}_{\mathrm{G}} \mathrm{R}} & 0 & 0 \\
0 & 0 & \frac{-1}{\mathrm{~T}_{\mathrm{P}}} & \frac{\mathrm{K}_{\mathrm{P}}}{\mathrm{T}_{\mathrm{P}}} & 0 \\
0 & 0 & 0 & \frac{-1}{\mathrm{~T}_{\mathrm{ch}}} & \frac{1}{\mathrm{~T}_{\mathrm{ch}}} \\
0 & \frac{1}{\mathrm{~T}_{\mathrm{rh}}} \frac{-\mathrm{F}_{1}}{\mathrm{~T}_{\mathrm{G}}} & \frac{-\mathrm{F}_{1}}{\mathrm{~T}_{\mathrm{G}} \mathrm{R}} & 0 & \frac{-1}{\mathrm{~T}_{\mathrm{rh}}}
\end{array}\right] \\
& \mathrm{B}=\left[\begin{array}{lllll}
0 & \frac{1}{\mathrm{~T}_{\mathrm{G}}} & 0 & 0 & \frac{\mathrm{F}_{1}}{\mathrm{~T}_{\mathrm{G}}}
\end{array}\right]^{\mathrm{T}}
\end{aligned}
$$

$\Gamma=\left[\begin{array}{lllll}0 & 0 & \frac{-K_{p}}{T_{p}} & 0 & 0\end{array}\right]^{\mathrm{T}}$

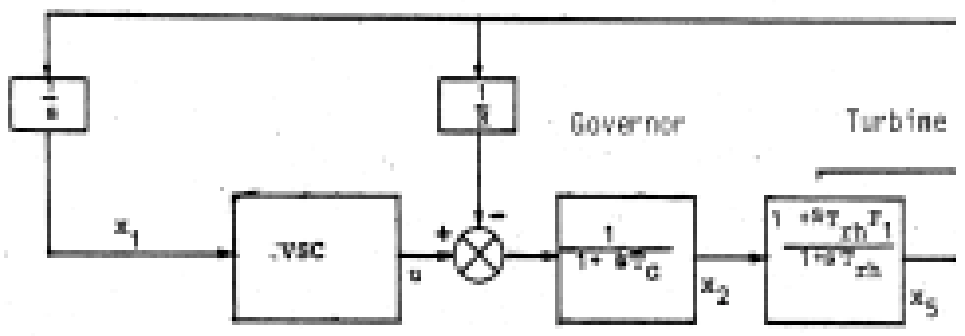

Fig. 1 Block Diagram os Steam - Dominated Area with VSC (67) 
The authors of reference [2] presented model modifications for integral control and that of linear optimal controller with which they compared their results. Since their results were found superior to the other two, it is only necessary here to compare the latter with those obtained in this paper.

\section{Statement of the Problem}

The automatic generation control problem for an isolated single area power system model of the type used here is to retain the power balance in the system such that the frequency $f$ is maintained at its nominal value $f$. In other words, the AGC scheme should generate an actuating signal $\mathrm{u}$ which acts on the speed changer position $\mathrm{P}_{\mathrm{c}}$ to adjust the valve opening to release the desired quantity of steam in response to unpredictable changes in load demand. However the resulting system transients must satisfy certain practically acceptable performance measures. These measures can be quantitatively assessed in terms of the overshoot, rise time and settling time of the frequency deviation and generation change transients, which result from a given magnitude of load demand variation.

\section{Variable Structure Unit Vector Controller Synthesis}

The essential feature of a variable structure control system (VSCS) is that the generally nonlinear system is provided with a means of automatically switching from one control structure to another according to a prescribed switching logic. A variable structure control system (VSCS) may, therefore, be regarded as consisting of a set of continuous subsystems each operating in a given subspace of the entire state space. The combination of these structures via a predetermined switching logic ensures the exploitation of the useful properties of each of the structures and possible creation of new properties not present in any of the structures. One such property is sliding motion, which occurs when the system state is constrained to move along the switching hypersurface. If the control form selected is such that this motion occurs on the intersection of all the switching hypersurfaces, the system is said to be in the sliding mode.

The two step design approach mentioned earlier will be implemented as follows:

\section{Solving The Existence Problem}

Consider the nominally linear system without disturbances given by

$$
\mathrm{X}(\mathrm{t})=\mathrm{AX}+\mathrm{bu}
$$

Where $b$ is an n-column vector and define a similarity transformation [7]

$$
\mathrm{Y}=\mathrm{MX}(\mathrm{t})
$$

Such that

$$
\mathrm{Mb}=\left[\begin{array}{l}
0 \\
\mathrm{~b}_{\mathrm{n}}
\end{array}\right]
$$

where $\mathrm{M}$ is $\mathrm{n} \times \mathrm{n}$ nonsingular matrix and $\mathrm{b}_{\mathrm{n}}$ is a nonzero element. Then from (4) and (5)

$$
\hat{y}=\mathrm{MAM}^{-1} \mathbf{y}+\mathrm{Mbu}
$$

Equation (7) can be written in the form

$$
\left[\begin{array}{l}
y_{1}^{\prime} \\
y_{2}^{\prime}
\end{array}\right]=\left[\begin{array}{ll}
\mathrm{A}_{11} & \mathrm{~A}_{12} \\
\mathrm{~A}_{21} & \mathrm{~A}_{22}
\end{array}\right]\left[\begin{array}{l}
y_{1}^{\prime} \\
y^{\prime}{ }_{2}
\end{array}\right]+\left[\begin{array}{c}
0 \\
\mathrm{~b}_{\mathrm{n}}
\end{array}\right] \mathrm{u}
$$

Where $A_{11}, A_{12}, A_{21}, A_{22}$ are respectively $(n-1) \times(n-1)$; $(\mathrm{n}-1) \times 1 ; 1 \times(\mathrm{n}-1) ; 1 \times 1$ submatrices. The motion of the system in sliding mode can then be specified by

$$
\begin{aligned}
& \dot{y}=\mathrm{A}_{11} \mathrm{y}_{1}+\mathrm{A}_{12} \mathrm{y}_{2} \\
& \sigma(\mathrm{y})=\mathrm{C}_{11} \mathrm{y}_{1}+\mathrm{C}_{12} \mathrm{y}_{2}=0
\end{aligned}
$$

where $C_{11}$ and $C_{12}$ are respectively $1 \times(n-1)$ and $1 \times 1$ satisfying the relation:

$$
\left[\begin{array}{ll}
\mathrm{C}_{11} & \mathrm{C}_{12}
\end{array}\right]=\mathrm{C}^{\mathrm{T}} \mathrm{M}^{-1}
$$

Rewriting equation (10) in the form,

$$
\mathrm{y}_{2}=-\mathrm{C}_{12}{ }^{-1} \mathrm{C}_{11} \mathrm{y}_{1}=-\mathrm{Fy}_{1}
$$

It follows that the motion of $y_{2}(t)$ in the sliding mode is linearly related to that of $y_{1}$, over the intersection of the switching hyperplane.

$$
\sigma(\mathrm{X})=\mathrm{CX}(\mathrm{t})=0
$$

Equation (9) and (12), which define the sliding motion, are structurally similar to the linear regulator problem with $\mathrm{y}_{2}$ playing the role of the control function and $y_{1}$ that of state vector. The "closed loop" system may be written as

$$
\dot{y}_{1}(t)=\left(A_{11}-A_{12} F\right) y_{1}(t)
$$

which shows that the design of a stable sliding mode $(\mathrm{y} \rightarrow 0$ as $\mathrm{t} \rightarrow$ :) with prescribed transient behaviour requires the determination of the gain matrix $F$ such that the matrix $\left(A_{11}\right.$ $\left.-A_{12} F\right)$ has prescribed (n-m) left hand plane eigenvalues. 
The matrix $\mathrm{F}$ can therefore be determined by using the well-known poleplacement design procedure. Having obtained $\mathrm{F}$ the elements of the hyperplane matrix $\mathrm{C}$ can be obtained by solving the equation [7].

$$
\mathrm{C}=\left[\mathrm{F}: \mathrm{I}_{\mathrm{m}}\right] \mathrm{M}
$$

Where $\mathrm{C}_{12}$ has been equated to unity without loss of generality.

\section{The Design of the Control Function}

The task here is to solve the reachability problem of selecting a state feedback control function $\mathrm{u}: \mathfrak{R}^{\mathrm{n}} \rightarrow \mathfrak{R}^{\mathrm{m}}$ which will drive the state $\mathrm{x}(\mathrm{t})$ from any initial condition $\mathrm{x}$ $(\mathrm{t})$ to the sliding manifold $\mathrm{CX}(\mathrm{t})=0$ and thereafter maintain it within this manifold towards the origin of coordinates i.e. $x(0)=0$. From the theory of vector spaces, it is evident that the sliding manifold is coincident with the null - space of matrix $\mathrm{C}$, written as $\mathrm{N}(\mathrm{C})$.

The general form of the variable structure control law consists of two parts: a linear part $\mathrm{u}_{\mathrm{L}}$ and a non- linear component $\mathrm{u}_{\mathrm{N}}$ which are added to form $\mathrm{u}$. The linear component of the control law is a state dependent feedback controller while the nonlinear part is discontinuous with respect to the state $\mathrm{x}(\mathrm{t})$ and incorporates all the nonlinear elements of the control law. $[9,10]$

In the existing variable structure control scheme for automatic generation control described in $[2,3,4]$, the nonlinear part of the control law is discontinuous on the individual hyperplanes, with the result that sliding conditions are separately derived for each switching surface. This often leads to waste of control efforts and an unnecessary enlargement of the control structure.

The proposed control function is based on a design philosophy that the individual controls are continuos except on the final intersection $\{\mathrm{N}(\mathrm{C})\}$ of the switching hyperplanes, where all the controls are discontinuous together. This approach ensures that the system motion is always towards the final target $\{\mathrm{N}(\mathrm{C})\}$, with the advantage that the control has a simpler structure and therefore, easier to implement. In other words, the proposed approach provides a systematic method for determining the linear $\left(\mathrm{u}_{\mathrm{L}}=\mathrm{Lx}(\mathrm{t}): \mathrm{L} \varepsilon \mathfrak{R}^{\mathrm{mxn}}\right)$ and non - linear $\mathrm{u}_{\mathrm{N}}$ components of an overall control structure $\left(\mathrm{u}=\mathrm{u}_{\mathrm{L}}+\mathrm{u}_{\mathrm{N}}\right)$ such that the nominally linearly controlled system has an (n- m) dimensional asymptotically stable manifold $\sigma=\mathrm{Cx}(\mathrm{t})$, on which motion is governed by a (n-m) - dimensional linear system with a prescribed eigenstructure spectrum. This motion must be preserved by the overall non-linearly controlled system. In the terminology of VSS theory, the proposed approach provides a feedback control structure, which guarantees the global reaching of the sliding mode and the preservation of motion on the sliding plane towards the origin of coordinates.

Following the general concept of unit vector control and considering the nominal linear system without disturbances (eqn. .4), the nonlinear component of the proposed control law is defined as:

$$
\mathrm{U}_{\mathrm{N}}=\frac{\rho}{\|\mathrm{Hx}(\mathrm{t})\|} \mathrm{Gx}(\mathrm{t}),(\rho>0)
$$

such that the overall control law is given by

$$
\mathrm{u}=\mathrm{U}_{\mathrm{L}}+\mathrm{U}_{\mathrm{N}}=\mathrm{Lx}(\mathrm{t})+\frac{\rho}{\|\mathrm{Hx}(\mathrm{t})\|} \mathrm{Gx}(\mathrm{t})
$$

Where $\mathrm{L}, \mathrm{G} \varepsilon \mathfrak{R}^{\mathrm{mxn}}$ and $\mathrm{H} \varepsilon \mathfrak{R}^{\mathrm{mxn}}$ are constant matrices such that $\mathrm{Cx}(\mathrm{t}) \equiv \mathrm{Gx}(\mathrm{t}) \equiv \mathrm{Hx}(\mathrm{t}) \equiv 0$ (i.e. the null spaces $\mathrm{N}(\mathrm{C}), \mathrm{N}$ $(\mathrm{G})$ and $\mathrm{N}(\mathrm{H})$ are coincident ) and $\rho>0$ is a constant parameter. The elements of matrices $\mathrm{L}, \mathrm{G}$ and $\mathrm{H}$ are determined as follows: from the transformed state $y$, [eqn. (5)], a second transformation $M_{2}: \mathfrak{R}^{\mathrm{n}} \rightarrow \mathfrak{R}^{\mathrm{n}}$ is formed such $\mathrm{M}_{2} \overline{\mathrm{Z}}=\left[\begin{array}{c|c}\mathrm{I}_{\mathrm{n}}-\mathrm{m} & 0 \\ \hline \mathrm{Mpy}_{\mathrm{p}} & \mathrm{Im}_{\mathrm{m}}\end{array}\right]$

Where $Z \& \Re^{\mathrm{n}}$ and

The matrix $\mathrm{F}$ is as defined in eqn. (12), while I stands for a unit matrix. Clearly the matrix $\mathrm{M}_{2}$ is non - singular and has the inverse

$\mathrm{M}_{2}^{-1}=\left[\begin{array}{c|c}\mathrm{In}-\mathrm{m} & 0 \\ \hline-\mathrm{F} & \mathrm{Im}\end{array}\right]$

If the transformed state variable $Z$ in eqn. (18) is partitioned such that

$Z^{\mathrm{T}}=\left[\begin{array}{ll}Z_{1} & Z_{2}\end{array}\right]^{\mathrm{T}}$

Where $Z_{1} \varepsilon \mathfrak{R}^{\mathrm{n} \_}{ }^{\mathrm{m}}$ and $Z_{2} \varepsilon \mathfrak{R}^{\mathrm{m}}$, then, substituting a partitioned $\mathrm{y}^{\mathrm{T}}$ into eqn.(18) and simplifying, yields

$$
\begin{aligned}
& \mathrm{Z}_{1}=\mathrm{y}_{1} \\
& \mathrm{Z}_{2}=\mathrm{Fy}_{1}+\mathrm{y}_{2}
\end{aligned}
$$

By comparing equations (21b) and (10) it is easily seen 
that the condition $Z_{2}=0$ and $\sigma=0$ are equivalent, in the sense that the points where $Z_{2}$ equals zero are precisely the same points where the original sliding manifold occurs. Now, differentiating eqns. (10) with respect to time and eliminating the y variables by making use of eqn. (8) and (21) gives the transformed state equations in terms of $Z$ as

$$
\begin{aligned}
& \dot{Z}_{1}=\Phi_{1} Z_{1}+A_{12} Z_{2} \\
& \dot{Z}_{2}=\Phi_{2} Z_{1}+\Phi_{3} Z_{2}+b_{11} 1
\end{aligned}
$$

Where

$\Phi_{1}=\mathrm{A}_{11}-\mathrm{A}_{12} \mathrm{~F} ; \Phi_{2}=\mathrm{F} \Phi_{1}-\mathrm{A}_{22} \mathrm{~F}+\mathrm{A}_{21} ; \Phi_{3}=\mathrm{A}_{22}+\mathrm{FA}_{12}$

For the nominal linearly controlled system $\mathrm{x}(\mathrm{t})=(\mathrm{A}-\mathrm{bL}) \mathrm{x}$ (t) to have an asymptotically stable sliding manifold, the linear component of the control law $\mathrm{u}_{\mathrm{L}}=\mathrm{Lx}(\mathrm{t})$ must be chosen so as to force $Z_{2}$ and to become identically zero. A simple way to achieve this is to equate eqn. (22b) to zero and solve for $u$ such that:

$\mathrm{u}_{\mathrm{o}}=-\mathrm{b}_{\mathrm{n}}{ }^{1}\left[\Phi_{2} \mathrm{Z}_{1}+\mathrm{f}_{3} \mathrm{Z}_{2}\right]$

However, to ensure that the motion of $\dot{Z}_{2}$ is asymptotically stable, the coefficient of $Z_{2}$ is modified so that, $u_{L}$ is chosen as

$\mathrm{u}_{\mathrm{L}}=-\mathrm{b}_{\mathrm{n}}{ }^{-1}\left[\Phi_{2} \mathrm{Z}_{1}+\left(\Phi_{3}-\Phi\right) \mathrm{Z}_{2}\right]$

Where $\Phi$ is any mxm matrix with left-hand half-plane eigenvalues. In this case, if a set of $m$ eigenvalues $\left(\lambda_{\mathrm{l}}, \mathrm{i}=1\right.$, $\mathrm{m})$ have been selected such that $\left[\lambda_{i}: \operatorname{Re}\left(\lambda_{i}\right)^{\circ} 0, i=1, \ldots \mathrm{m}\right]$, then $\phi$ may be chosen as $\phi=\operatorname{diag}\left[\lambda_{i}, \mathrm{i}=1, \ldots \mathrm{m}\right]$. Transforming back into original state space gives

$\mathrm{L}=-\mathrm{b}_{\mathrm{n}}-{ }^{1}\left[\begin{array}{ll}\Phi_{2} & \Phi_{3}-\Phi\end{array}\right] \mathrm{M}_{2} \mathrm{M}$

The linear control law only serves to drive the transformed state component $Z_{2}$ to zero. For the state function $Z(t)$ to attain the sliding manifold $N(C)$ in finite time, the discontinuous control law is required. The only restriction in the choice of $u_{\mathrm{N}}$ is that $\mathrm{u}_{\mathrm{N}}$ should be zero only when $\mathrm{Z}_{2}$ $=0$, at which time it switches to another preselected value. In other words, the discontinuous control law should be selected such that the hyperplane $Z_{2}=0$ serves as the switching surface, on which sliding motion occurs. By definition $\mathrm{u}_{\mathrm{N}}$ is continuous everywhere on the state space except on the sliding manifold where $Z_{2}=0$. Thus, letting $\mathrm{p}_{\mathrm{n}}$ denote the positive - definite solution of the Lyapunov equation

$\mathrm{P}_{\mathrm{n}} \Phi+\Phi^{\mathrm{T}} \mathrm{P}_{\mathrm{n}}+\mathrm{I}_{\mathrm{m}}=0$
Then $P_{n} Z_{2}=0$ if and only if $Z_{2}=0$. The discontinuous control law is, therefore, chosen as

$\mathrm{U}_{\mathrm{N}}(\mathrm{Z})=\frac{2 \rho}{\left.\| \mathrm{P}_{\mathrm{n}} \mathrm{Z}_{2}\right) \|} \mathrm{b}_{\mathrm{n}}^{-1} \mathrm{P}_{\mathrm{n}} \mathrm{Z}_{2}\left(\mathrm{Z}_{2} \neq 0\right)$

Where $\rho>0$ is a scalar parameter to be selected by the designer. When $Z_{2}=0, \mathrm{u}_{\mathrm{N}}$ is arbitrarily chosen as a function satisfying:

$$
\left\|\mathrm{u}_{\mathrm{N}}\right\| \leq \rho
$$

This choice has the advantage of limiting the switching time. Large switching time is known to be responsible for the chattering motion associated wit variable structure systems in the sliding mode. Expressing the discontinuous control submatrices eqn. (17) in the $\mathrm{x}$-space yields

$$
\begin{aligned}
& \mathrm{G}=-\mathrm{B}_{2}-{ }^{1}\left[\begin{array}{ll}
0 & \mathrm{P}_{\mathrm{n}}
\end{array}\right] \mathrm{M}_{2} \mathrm{M} \\
& \text { and } \\
& \mathrm{H}=\left[\begin{array}{ll}
0 & \mathrm{P}_{\mathrm{n}}
\end{array}\right] \mathrm{M}_{2} \mathrm{M}
\end{aligned}
$$

\section{Application Example}

For the system model used in [2], the parameter values are given as

$\mathrm{T}_{\mathrm{G}}=0.08 \mathrm{secs} \quad \mathrm{T}_{\mathrm{rh}}=10 \mathrm{secs} \quad \mathrm{K}_{\mathrm{p}}=120 \mathrm{~Hz} / \mathrm{p}$. u. Mw

$\mathrm{T}_{\mathrm{ch}}=0.3 \operatorname{secs} \quad \mathrm{T}_{\mathrm{P}}=20 \operatorname{secs} \quad \mathrm{F}_{1}=0.5$

$\mathrm{R}=2.4 \mathrm{~Hz} / \mathrm{p} . \mathrm{u} \cdot \mathrm{Mw}$

Substituting for the above data in eqn. (3) yields the following system matrices:

$\mathrm{A}=$

$\left[\begin{array}{ccccc}0 & 0 & 1 & 0 & 0 \\ 0 & -12.5 & -5.208 & 0 & 0 \\ 0 & 0 & -0.05 & 6 & 0 \\ 0 & 0 & 0 & -3.33 & 3.33 \\ 0 & -6.15 & -2.604 & 0 & -0.1\end{array}\right]$
$\mathrm{B}=[0$
$12.5 \quad 0 \quad 0$
$6.25]^{\mathrm{T}}$
$\Gamma=\left[\begin{array}{lllll}0 & 0 & -6 & 0 & 0\end{array}\right]^{\mathrm{T}}$

Where superscript $\mathrm{T}$ denotes vector or matrix transpose

The $\mathrm{n} \times \mathrm{n}$, orthogonal transformation matrix $\mathrm{M}$ is chosen as: 
$\mathrm{M}=\left[\begin{array}{ccccc}1 & 0 & 0 & 0 & 0 \\ 0 & 1 & 0 & 0 & -2 \\ 0 & 0 & 1 & 0 & 0 \\ 0 & 0 & 0 & 1 & 0 \\ 0 & 0 & 0 & 0 & 1\end{array}\right]$

such that eqn. (2) is satisfied, with the result that the transformed system matrix become

$\left[\begin{array}{c|c}\mathrm{A}_{11} & \mathrm{~A}_{12} \\ \hline \mathrm{A}_{21} & \mathrm{~A}_{22}\end{array}\right]=\left[\begin{array}{cccc|c}0 & 0 & 1 & 0 & 0 \\ 0 & -.02 & 0 & 0 & -0.2 \\ 0 & 0 & -.05 & 6 & 0 \\ 0 & 0 & 0 & -3.33 & 3.33 \\ \hline 0 & -6.15 & -2.604 & 0 & -0.1\end{array}\right]$

By fixing the eingenvalues of the reduced order closed loop system of eqn. (15) at $-3,-5,-7 \pm \mathrm{j} 5$ and following the procedure outlined earlier, the $(n-m) \times m$ order matrix $F$ is obtained as,

$\mathrm{F}=\left[\begin{array}{llll}5.72 & -2.25 & 5.80 & 2.92\end{array}\right]$

Then, from eqn. (15) the hyperplane matrix $\mathrm{C}$ is computed to yield,

$$
\mathrm{C}=\left[\begin{array}{lllll}
5.72 & -2.25 & 5.80 & 1.0 & 2.92
\end{array}\right]
$$

In order to synthesize the unit vector control function, a second transformation matrix which transforms the variable $\mathrm{y}(\mathrm{t})$ to $\mathrm{z}(\mathrm{t})$ in eqn. (18) is chosen as,

$$
\mathrm{M}_{2}=\left[\begin{array}{l|l}
\mathrm{I} & 0 \\
\hline \mathrm{F} & \mathrm{I}_{1}
\end{array}\right]=\left[\begin{array}{ccccc}
1 & 0 & 0 & 0 & 0 \\
0 & 1 & 0 & 0 & 0 \\
0 & 0 & 1 & 0 & 0 \\
0 & 0 & 0 & 1 & 0 \\
5.72 & -2.25 & 5.80 & 2.92 & 1
\end{array}\right]
$$

Such that

$$
\mathrm{M}_{2}^{-1}=\left[\begin{array}{ccccc}
1 & 0 & 0 & 0 & 0 \\
0 & 1 & 0 & 0 & 0 \\
0 & 0 & 1 & 0 & 0 \\
0 & 0 & 0 & 1 & 0 \\
5.72 & -2.25 & 5.80 & 2.92 & 1
\end{array}\right]
$$

Then, the coefficients $\phi_{1}, \phi_{2}, \phi_{3}, \mathrm{~A}_{12}$ and $\mathrm{B}_{2}$ in eqn. (22) become

$$
\phi_{1}=\left[\begin{array}{cccc}
0 & 0 & 1 & 0 \\
0 & -0.2 & 0 & 0 \\
0 & 0 & -0.05 & 6 \\
0 & 0 & 0 & -3.33
\end{array}\right]
$$

$\phi_{2}=\left[\begin{array}{llll}10.75 & -10.72 & 15.76 & 31.62\end{array}\right]$

$$
\begin{aligned}
& \phi_{3}=[-2.23] \\
& \mathrm{B}_{2}=[6.25]
\end{aligned}
$$

Since the system under consideration is single - input single - output (SISO), matrix $\phi$ in eqn. (25) may be taken as any arbitrary negative scalar number. By choosing $\phi=-$ 1 , the linear component of the control law defined by eqn. (25) reduces to

$$
\mathrm{L}=\left[\begin{array}{lllll}
-0.58 & 1.27 & -1.09 & -4.48 & -2.54
\end{array}\right]
$$

In order to determine the nonlinear part of the proposed control law, the Lyapunov equation given by eqn. (26) must first be solved for the positive definite matrix $\mathrm{P}_{n}$. However, for a SISO system, the matrix $\mathrm{P}_{\mathrm{n}}$ becomes a scalar. Substituting $\phi=-1$ in eqn. (26) yields:

or

$$
-P_{n}-P_{n}+1=0
$$

$$
\mathrm{P}_{\mathrm{n}}=0.5
$$

Then, eqns.(29) and (30) give the values of $\mathrm{G}$ and $\mathrm{H}$ respectively as

$\mathrm{G}=\left[\begin{array}{lllll}-0.46 & 0.18 & -0.46 & -0.23 & -0.44\end{array}\right]$

$\mathrm{H}=\left[\begin{array}{lllll}2.86 & -1.13 & 2.9 & 1.46 & 2.75\end{array}\right]$

The values of the parameter $\rho$ is left for the designer to choose. However, to ensure numerical stability, the positive constant $\rho$ should be of the order of ten or lower. In the present case, the value of $\rho$ is fixed at 5.0. Now, substituting for the values L, G, H and $\rho$ obtained above, into eqn. (17) gives

$\mathrm{UL}_{\mathrm{L}}=\left[\begin{array}{lllll}-0.58 & 1.27 & -1.09 & -4.48 & -2.54\end{array}\right] \mathrm{x}(\mathrm{t})$

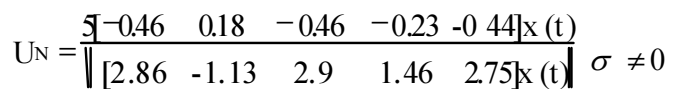

or $\mathrm{u}_{\mathrm{N}}=3 ; \sigma=0$ 


\section{Simulation Results}

Experimentation with models has become a powerful tool for predicating the behavior of designed systems under envisaged working conditions even before actual construction commences. This is because, in quite a number of cases, simulation has been found to be faster and more cost-effective than experimenting with physical components.

In this subsection, the simulation of the performance of proposed VSAGC scheme under various working conditions is achieved by solving eqn. (1) using a fourth order Runge Kutta integration algorithm. However, the variable $\mathrm{u}(\mathrm{t})$ is replaced by the variable structure control scheme of eqn. (31), while $\xi(t)$ is taken to be a step change in load demand, which results in frequency fluctuation about the nominal value.

For the sake of comparison, the performance of the VSAGC strategy proposed by Chan and Hsu [2] is also presented. In this case, the hyperplane matrix $\mathrm{C}$ is given by

$$
\mathrm{C}=\left[\begin{array}{lllll}
100 & -0.0355 & 11.7 & 11 & 1.07
\end{array}\right]
$$

The control function is in the form mentioned earlier with the result that the control parameters are given as;

$$
\begin{aligned}
& \psi_{1}= \begin{cases}\alpha_{1}=100 & \text { if } x_{1} \sigma>0 \\
\beta_{1}=-100 & \text { if } x_{1} \sigma<0\end{cases} \\
& \psi_{3}=\left\{\begin{array}{l}
\alpha_{3}=100 \text { if } x_{3} \sigma>0 \\
\beta_{3}=-100 \text { if } x_{3} \sigma<0
\end{array}\right. \\
& \psi_{2}=\psi_{4}=\psi_{5}=0
\end{aligned}
$$

For ease of comparison, the curves showing the performance of the proposed VSAGC scheme are superimposed on the curves showing the response of the existing strategy to the same change in load. Figures 2 to 4 depict the response of both systems to step changes in load demand of magnitudes $0.01,0.06$ and $0.1 \mathrm{pu}$ respectively. In fig. 2 representing normal system operation, whereby load changes are not expected to vary beyond $0.01 \mathrm{pu}$, the response of both systems are acceptable. However, the proposed scheme shows superior performance, in the sense that the existing scheme exhibits higher frequency deviation and longer settling time for the transient generation change. Specifically, the transient response of the change in power generation settled at 1.6 seconds for the proposed scheme, and 5.2 seconds for the existing scheme.
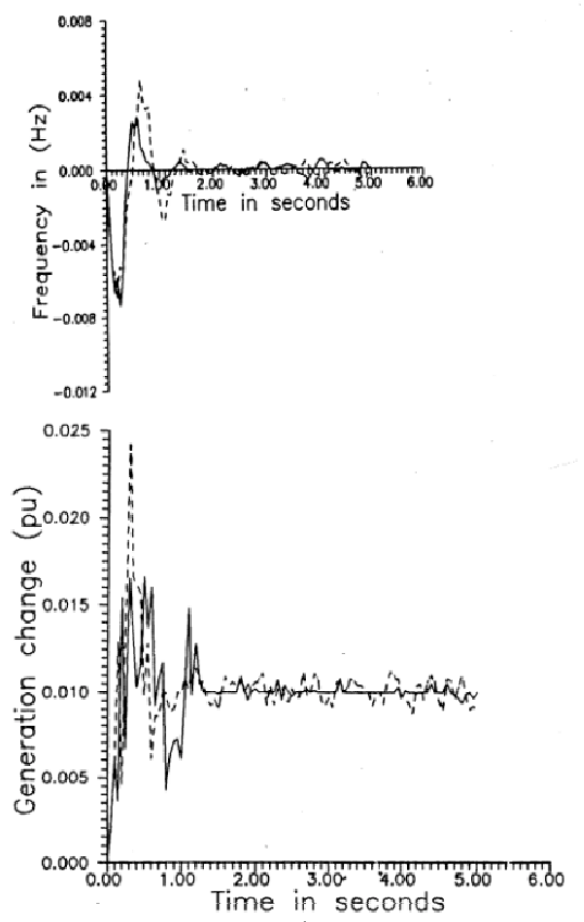

Fig. 2: System Responses for 0.01 pu Step Chamge on Load (a) Frequency Change (b) Generation Change Proposed Scheme -.---- Existing Scheme

The superiority of the proposed scheme becomes clearer as the magnitude of the load demand grows higher. For $\Delta \mathrm{P}_{\mathrm{D}}=0.06 \mathrm{pu}$, (Fig.3) representing moderate abnormal situation, the frequency transient of both schemes still remain acceptable, though the response of the existing scheme is characterised by relatively higher overshoots. However the transient of the generated power remains for more than 10 seconds for the existing scheme while that of the proposed scheme settled after about 2 seconds. An even worse situation is depicted in fig. 4 for $\Delta P_{D}=0.1 \mathrm{pu}$ which represents a serious abnormal operating condition. Here both the frequency and generated power transients for the existing technique are unacceptable due to their continuous oscillation, which can trigger-off large amplitude swings of the entire power system. It is observed however, that the response of the proposed scheme still remained acceptable since the resulting vibrations due to the large change in load demand settled in about 2.5 seconds. 


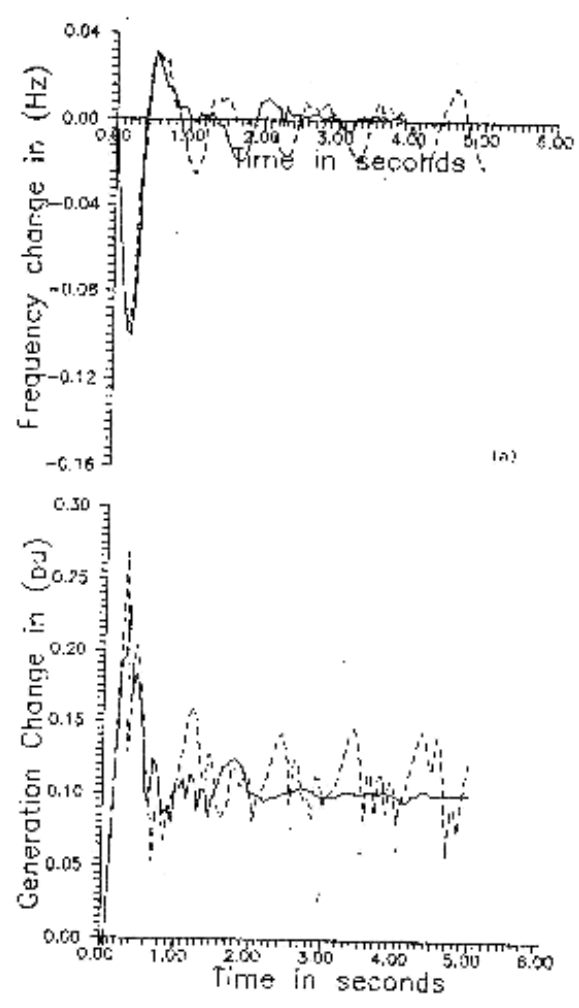

Fig. 3: System Responses for 0.06 pu Step Chamge on Load (a) Frequency Change (b) Generation Change Proposed Scheme

Existing Scheme
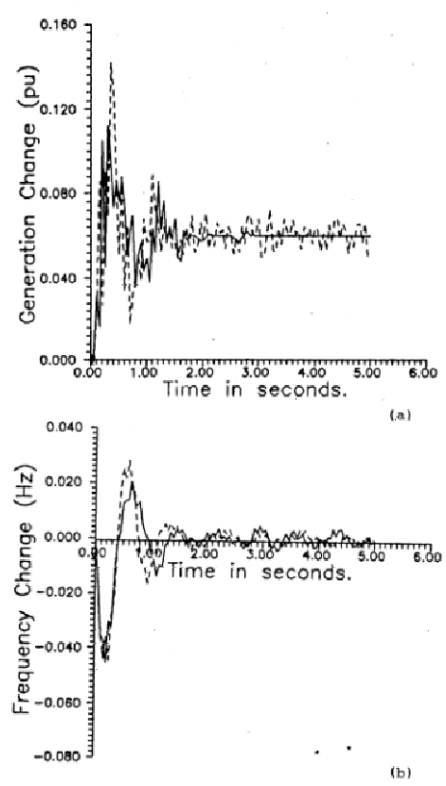

Fig. 4: System Responses for 0.1 pu Step Chamge on Load (a) Frequency Change (b) Generation Change

- Proposed Scheme Existing Scheme
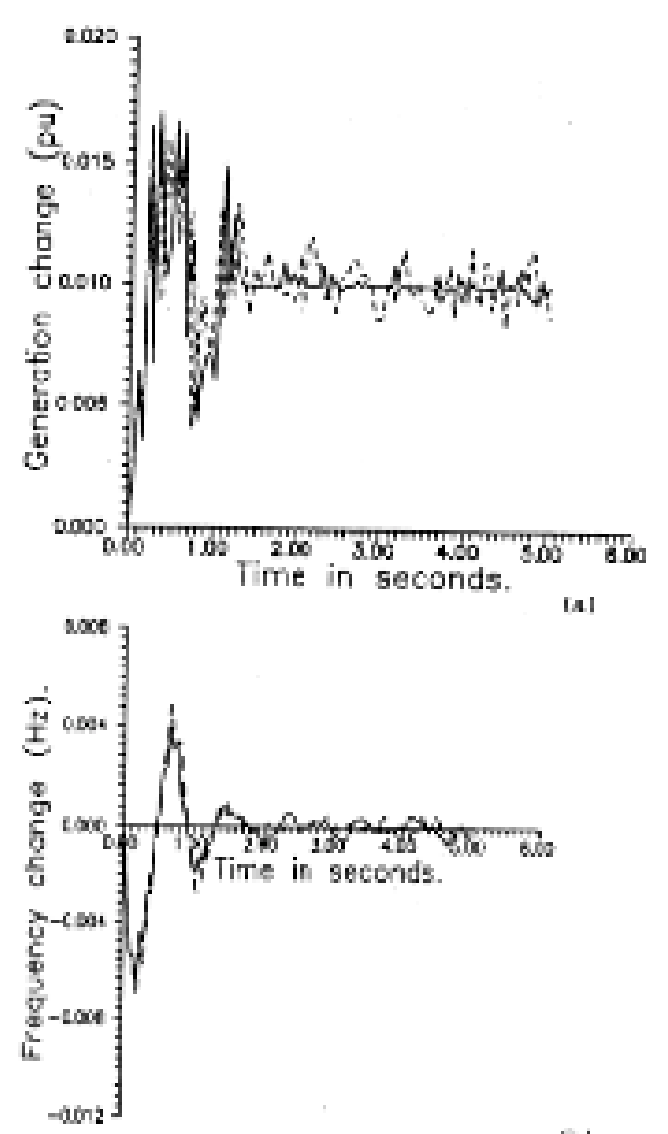

B!

Fig. 5: System Responses for 0.01 pu Step Chamge on Load with Generation Rate Constraint.

(a) Frequency Change (b) Generation Change

— Proposed Scheme --.--- Existing Scheme

\section{Generation Rate Constraints}

Now, the simulation results discussed above had assumed that the power system possesses the extra capacity to take up the load increase and there is no constraint on the rate at which the extra capacity is made available. In practical power systems however, there exsits a rate of change of generation which must be known. For power systems dominated by steam power plants, like the system under consideration, the rate of generation varies between 0.01 and 0.1 pu per minute [2]. Figure 5 depicts simulation results for a $0.01 \mathrm{pu}$ change in load demand where $0.1 \mathrm{pu}$ per minute rate of change of generation has been imposed. It is observed that the system transients still settled at about 3.5 seconds for the proposed scheme while it is not so for the existing strategy. Of particular importance is that the generated power transient for the existing VSAGC scheme persists for a long time. This has the potential danger of 
triggering wide system oscillations leading to possible system collapse. Such a situation is very undesirable.

\section{CONCLUSION}

This paper has presented a new systematic procedure, for the synthesis of variable structure automatic generation controllers. The proposed design scheme utilizes a particular canonical transformation, which decouples the state space and facilitates the easy determination of the sliding mode - condition. The equations of sliding motion so determined are seen to be structurally similar to the linear regulator problem which suggests that a modified form of the conventional eigenstructure assignment technique can be employed for the computation of the hyperplane matrix $\mathrm{C}$.

The synthesis of the proposed control function similarly follows a systematic procedure based on the unit vector control design approach. The resulting control function guarantees asymptotically stable sliding motion as well as ensures faster reaching of the sliding plane; in the sense that the frequency and generated power transients following a step load change settles faster than for the existing VASGC scheme. It also has the advantage of simpler structure since only one switching action is required as opposed to the control function for VSAGC so far used in the technical literature which has the form of relays with state dependent gains. In that case, the number of switching corresponds to the number of states, that is, the system order.

Finally, by employed a model of the power system commonly used in the technical literature, comparative simulation studies were performed to show the superiority of the proposed scheme.

\section{ACKNOWLEDGEMENTS}

The author gratefully acknowledges the financial support of the Central Research Committee of the University of Lagos in carrying out this work.

\section{REFERENCES}

Bengiamin, N. N. and Chan, W. C. "Variable Structure Control of Electric Power Generation" IEEE Trans. PAS -101, pp. 376-380, 1982.

Chan, W. C. and Hsu, Y. Y. "Optimal Control of Electric Power Generation using Variable Structure Controllers" Electr. Power Syst. Res. Vol. 6, pp. 269 $278,1983$.
- Ibid - "Automatic Generation Control of Interconnected Power Systems using Variable Structure Controllers" Proc. IEE, vol. 128, pt.C, No. 5, Sept. 1981, pp.269279.

Sivaramakrishnan, A. Y. et al. "Design of Variable-Structure Load - Frequency Controller using Pole Assignment Technique” Int. J. Contr. 40, No. 3, pp. 487-498, 1984.

Itkins, U. "Control Systems of Variable Structure" Wiley, New York 1976.

Utkin, V. I. "Sliding Modes and their Application in Variable Structure Systems", IEEE Trans. Vol. AC 22, No. 2, April 1977.

Utkin, V. I. and K. D. Yang, "Methods for Constructing Discontinuity Planes in VSS" Translated from Automatika i Telemekhanika, No. 10, pp. 72 - 77, October 1978.

Dorling, C. M. and Zinober, A. S. I. "Two Approaches to Hyperplane Design in Multivariable Variable Structure Control Systems". INT. J. Control, 1986, vol. 44, No. 1, $65-82$.

Utkin, V. I. "Discontinuous Control Systems: The state of the Art in Theory and Practice" IFAC Congress, Munich, 1987, pp. 75 - 94.

Ryan, E. P. and Coreless, M. "Ultimate Boundedness and Asymptotic Stability of a Class of Uncertain Systems". IMA Journal of Math, Control Information; vol. 1, No. 3, pp. $223-242,1984$. 